\title{
La bande dessinée, un objet didactique mal identifié
}

\section{Marianne Blanchard et Hélène Raux}

\section{(2) OpenEdition}

1 Journals

Édition électronique

URL : http://journals.openedition.org/trema/4818

DOI : 10.4000/trema.4818

ISSN : 2107-0997

Éditeur

Faculté d'Éducation de l'université de Montpellier

Édition imprimée

ISBN : 979-10-96627-07-3

ISSN : 1167-315X

Référence électronique

Marianne Blanchard et Hélène Raux, "La bande dessinée, un objet didactique mal identifié ", Tréma [En ligne], 51 | 2019, mis en ligne le 01 avril 2019, consulté le 05 août 2019. URL : http:// journals.openedition.org/trema/4818; DOI : 10.4000/trema.4818

Ce document a été généré automatiquement le 5 août 2019

Trema 


\title{
La bande dessinée, un objet didactique mal identifié
}

\author{
Marianne Blanchard et Hélène Raux
}

1 De l'école à l'université, l'idée que la bande dessinée a sa place dans un contexte d'enseignement semble avoir fait son chemin en France : des bandes dessinées figurent parmi les ouvrages recommandés pour l'enseignement de la littérature à l'école élémentaire depuis 2002, le festival de la bande dessinée d'Angoulême collabore autour de différents projets (concours de BD scolaires, prix des écoles/collèges/lycées) avec le Ministère de l'Éducation nationale depuis 2007, les résidences d'auteurs de bande dessinée en milieu scolaire se multiplient, etc.

2 Pour autant, la légitimité scolaire de la bande dessinée reste faible comparativement à d'autres supports, en témoigne sa place encore restreinte dans les programmes : Aquatias (2017) note par exemple qu'en français la bande dessinée est mentionnée parmi les genres à lire à l'école et au collège mais conserve une place très marginale, tandis qu'en arts plastiques, les programmes l'abordent plus souvent «en filigrane » qu'explicitement ${ }^{1}$. Dans ce contexte, il n'est guère étonnant que la bande dessinée reste peu mobilisée en milieu scolaire: les enquêtes réalisées sur les lectures à l'école indiquent ainsi qu'en littérature elle n'occupe qu'une place très marginale parmi les œuvres lues dans les classes (Louichon, 2008 ; Dardaillon, 2009 ; Bonnéry et al., 2015). De fait, c'est finalement hors des salles de classes que la bande dessinée semble être le plus lue en milieu scolaire, qu'elle soit sortie des cartables entre les cours, dans les "interstices des emplois du temps » (Détrez et Vanhée, 2012, p. 63) ou consultée dans les Centres de Documentation et d'Information (Depaire, 2019).

Cette faible présence en classe de la bande dessinée peut s'expliquer par l'image négative encore accolée à une partie de la production éditoriale dans ce domaine. En effet, bien que les observateurs s'accordent sur le processus de reconnaissance dont le « $9 \mathrm{e}$ art » a fait l'objet au cours des cinquante dernières années (Boltanski, 1975; Guilbert, 2011; Maigret, 2015; Groensteen, 2017), ils insistent également sur l'opposition qui s'est progressivement construite entre «BD cultivée et BD de masse » (Maigret, 1994) et que recoupent d'autres clivages : «bande dessinée enfantine vs bande dessinée pour adultes, 
empire du mainstream $v s$ hérauts de l'édition alternative, domaine des séries vs roman graphique» (Lesage, 2018). Comme l'indique J. M. Méon (2015), la légitimation de ce médium apparaît conditionnée par la « reprise de critères et de pratiques importés de domaines artistiques plus légitimes » : c'est Hergé en tant que «dessinateur » que l'on entend présenter au Centre Pompidou en 2006, Hugo Pratt comme « aquarelliste » que l'on veut faire découvrir à la Pinacothèque de Paris en 2011 ou encore Moebius comme artiste polyvalent "qui dépasse les limites de sa discipline" que l'on honore à la Fondation Cartier en 2010-2011; les créateurs et les œuvres sont valorisés à la condition qu'ils puissent être rattachés à des formes artistiques plus établies, plus « nobles ».

4 Ce «soupçon d'indignité » (Lesage, 2018) qui pèse sur une partie des œuvres de bande dessinée ne saurait pour autant expliquer à lui seul la faible présence de ce médium dans les salles de classes. On peut supposer que le manque de maitrise du support par les enseignants joue également un rôle: peu présente dans les programmes scolaires, la bande dessinée est quasiment absente des cursus universitaires, notamment dans les composantes chargées de la formation des enseignants (ESPE) - même si elle peut être abordée ponctuellement par des enseignants-chercheurs impliqués dans des recherches sur le médium, qui connaissent actuellement un important développement dans une diversité de disciplines (Berthou, 2015; Baudry, 2015). Au cours de leur formation, les rares enseignants qui découvrent la bande dessinée comme objet d'étude ne le doivent qu'au hasard de rencontres avec des universitaires engagés dans des travaux dans le domaine ou d'initiatives locales, en particulier dans les environs d'Angoulême, où la Cité internationale de la bande dessinée et de l'image structure une offre de formation à destination des enseignants. Mais pour la plupart, ces derniers n'ont jamais reçu de formation relative à la bande dessinée et se disent démunis devant cet objet (Dardaillon, 2009) ${ }^{2}$. Cet embarras des enseignants face à l'« objet culturel non identifié » (Groensteen, 2006) qu'est la bande dessinée se traduit dans certains des usages éducatifs qui en sont faits, dans les propositions de manuels comme dans les pratiques effectives: de fait, lorsqu'elle n'est pas ignorée, la bande dessinée est souvent sous-utilisée, voire mal utilisée. Autrement dit, « après le temps du mépris vient celui de la méprise » (Rouvière, 2012, p. 11).

5 En premier lieu, la bande dessinée n'est pas toujours utilisée pour elle-même: «accroche » pour « déclencher " des situations de cours ou «marchepied» vers des œuvres plus complexes (Rouvière, 2017), elle n'est alors qu'un prétexte pour capter l'attention des élèves/étudiants et les conduire vers d'autres supports d'apprentissage. De la même façon, les bandes dessinées sont rarement étudiées comme des œuvres intégrales (Rouvière, 2017), et on retrouve souvent ce médium cantonné à un rôle d'illustration, par exemple pour dynamiser des exercices de grammaire (Bomel-Rainelli et Demarco, 2011), ou sous forme de vignette isolée au sein de dossiers documentaires dans une diversité de disciplines, comme en histoire où des vignettes apparaissent parfois dans les pages de manuels sans être questionnées dans leur statut de document (Mack dit Mack, 2012).

6 En second lieu, la bande dessinée est considérée comme facile d'accès, et donc comme facilitant nécessairement les apprentissages. Aussi est-elle souvent utilisée en classe avec le postulat selon lequel les élèvent sauraient d'emblée la lire. Pourtant, les données empiriques montrent que ce postulat est erroné. Plusieurs études menées au Québec avec des élèves à différents niveaux du cursus indiquent qu'ils peinent à acquérir à travers un support en bande dessinée des connaissances disciplinaires. En effet, la lecture de ce médium «multimodal» implique d'articuler deux modes sémiotiques spécifiques: le 
texte et l'image. Or les élèves s'appuient peu sur les éléments visuels pour extraire des informations d'une bande dessinée historique (Martel et Boutin, 2015) ou pour justifier l'interprétation d'un extrait de roman graphique (Lacelle, 2012). Les travaux s'attachant à décrire les compétences en jeu dans la «littératie multimodale» montrent ainsi l'importance de former les élèves à la lecture de ces supports complexes.

7 Loin de faciliter la compréhension et les apprentissages, l'usage de la bande dessinée peut même leur nuire, comme le montrent Bautier et al. (2012). Ces auteurs s'inscrivent dans un cadre théorique mettant en avant le rôle des « malentendus socio-cognitifs » dans les difficultés scolaires rencontrées par beaucoup d'élèves issus des familles populaires (Bautier et Rochex, 1997). Les élèves non familiarisés dans leur environnement familial avec les règles de l'univers scolaire auraient en effet tendance à se focaliser «sur la dimension visible des prescriptions enseignantes: accomplir un exercice, arriver au résultat, avoir une bonne note... sans conscience que ces tâches ne sont que le moyen pédagogique de comprendre ou de consolider un savoir, ce qui est rarement explicité » (Bonnery, 2007, p. 111) : devant une carte de géographie, l'élève dans le malentendu se focalise sur l'activité de coloriage au détriment des apprentissages visés (savoir lire ou produire une légende, mémoriser les informations codées par les couleurs, etc.). Or l'usage de la bande dessinée en classe peut participer de la production de ces malentendus, si elle est perçue comme un simple vecteur de motivation censé faciliter l'accès à des apprentissages, sans que ceux-ci soient clairement explicités : Rouvière pointe par exemple dans le dossier comment devant une banque d'images, les élèves peuvent être absorbés par le choix des éléments et la composition des vignettes les plus riches, et ainsi perdre de vue la cohérence du récit. En outre, Bautier et al. (2012) mettent en évidence, dans le cadre d'une séance de lecture en $\mathrm{CP}$, les risques liés à la sousestimation des difficultés suscités par un support en bande dessinée. Il s'agit d'un récit d'une page, constitué de sept vignettes présentant une diversité sémiotique importante (texte sous chaque vignette, phylactères, codes pour représenter les états mentaux du personnage principal, symboles graphiques exprimant un mouvement, etc.). Les élèves peinent de fait à lire le document, et sollicitent l'enseignant, chacun sur ses difficultés propres, contribuant ainsi à morceler la séance. Réciproquement, l'enseignant lui-même, face aux demandes des élèves, décompose le support «d'une manière telle que l'unité choisie pour focaliser l'attention des élèves (un mot, une phrase, une vignette, etc.) n'apparait plus comme faisant partie d'un ensemble, et devient valeur en soi ». Or ce « double morcellement » s'effectue au détriment de la possibilité pour tous les élèves de saisir la cohérence de la planche et de percevoir les enjeux de la séance. Loin de montrer que la bande dessinée n'a pas sa place à l'école, ces exemples rappellent la spécificité de ce médium et la nécessité de l'enseigner pour lui-même avant de pouvoir l'utiliser comme support pour d'autres apprentissages.

On peut enfin identifier une troisième "méprise " relative aux usages de la bande dessinée en classe : l'attrait supposé qu'elle susciterait chez les élèves/étudiants. L'intérêt des élèves pour le médium est en effet à nuancer. La vaste enquête nationale sur la lecture de bande dessinée en France réalisée en $2011^{3}$ montre ainsi que si $76 \%$ des 7-10 ans interrogés déclarent lire des bandes dessinées, cette proportion atteignant un pic (90\%) dans la tranche d'âge des 11-14 ans, le nombre de lecteurs de bande dessinée décroît ensuite fortement : ils ne sont plus que $50 \%$ chez les $15-17$ ans, et $35 \%$ chez les 18-24 ans. Chez les plus âgés, beaucoup déclarent être d'anciens lecteurs de bande dessinée ( $46 \%$ des 15-17 ans, $58 \%$ des 18-24). Cet abandon de la lecture de bande dessinée 
au fil des âges s'explique pour partie par un déclin plus général des pratiques de lecture : selon les données d'une enquête IPSOS conduite en 2016, 37 \% des enfants lisent tous les jours ou presque pour leur plaisir au primaire, contre $27 \%$ au collège et $19 \%$ au lycée (enquête citée par Aquatias, 2017). Mais, comme le souligne Sylvain Aquatias, « un autre facteur apparaît dans l'arrêt de la lecture de BD à l'adolescence ». Dans l'enquête de 2011, $62 \%$ des 15-17 ans anciens lecteurs de bande dessinée sont :

totalement d'accord ou plutôt d'accord avec le fait que les bande dessinées sont surtout faites pour les enfants et les jeunes. En fait, si cette conception est majoritaire chez ceux qui arrêtent la BD, elle est minoritaire chez ceux qui sont encore lecteurs (36\%).

9 Un nombre non négligeable de jeunes arrête donc de lire de la bande dessinée car ils pensent qu'elle n'est plus de leur âge. Or le fait d'utiliser la bande dessinée à des fins pédagogiques peut renforcer chez ces derniers la représentation d'un médium destiné aux enfants, les éloignant encore plus de cette lecture dont ils se détournent « pour bien montrer qu'ils ont grandi » (Aquatias, 2017 - voir aussi Aquatias, 2015). En outre, ceci rappelle que le choix des bandes dessinées étudiées en classe est particulièrement important, puisque des œuvres perçues comme « enfantines » par les élèves plus âgés et les étudiants pourraient susciter une forme de rejet, contraire à l'effet attractif espéré.

10 Ces méprises et ces mésusages de la bande dessinée persistent d'autant plus dans les représentations et les pratiques que rares sont les recherches susceptibles d'éclairer les enjeux des usages de la bande dessinée en contexte d'enseignement. Baudry (2015) note qu'une seule des 59 thèses soutenues ou en cours sur la bande dessinée en France entre 2010 et 2015 est inscrite en sciences de l'éducation (contre 12 en littérature et langue française ou 9 en langue et civilisation étrangère), tandis que Martel et Boutin (2015) déplorent le peu de recherches portant sur l'expérimentation en classe de ce medium. Rouvière (2017) rappelle quant à lui que les premiers colloques consacrés à la fin des années 1970 aux liens entre bande dessinée et éducation n'ont pas eu de suite immédiate, et qu'il a fallu attendre le colloque de 2008 à Rimouski (Québec) puis celui de 2010 à Grenoble "Lire et produire des BD à l'école» et les Actes "Bande dessinée et enseignement des humanités» pour qu'on se ressaisisse de la question. Dans cette situation, des initiatives émanent du champ de la bande dessinée plutôt que du monde scolaire pour fédérer acteurs du livre et de l'éducation autour d'une « réflexion collective sur la manière de promouvoir davantage la bande dessinée au sein des établissements ", objectif donné à une étude lancée par le Syndicat National des Editeurs et publiée en janvier 2019 (Depaire, 2019).

11 C'est avec ce double constat à l'esprit - sous, ou mésusage la bande dessinée dans les contextes d'enseignement d'une part, petit nombre de recherche consacrées spécifiquement aux usages pédagogiques et didactiques de ce médium d'autre part - que nous avons lancé l'appel à contribution pour le présent dossier.

12 Les auteurs étaient invités à réfléchir autour de trois axes: 1. La place de la bande dessinée dans les pratiques effectives d'enseignement (quels usages en classes? quels corpus ?) ; 2. La spécificité du médium (Quelle plus-value pédagogique peut être attendue de la lecture ou de la production de bande dessinée dans des situations d'enseignement? Et comment sa spécificité peut-elle être pleinement prise en compte?); 3. Les compétences des élèves/enseignants (A quelles conditions la bande dessinée peut-elle être mobilisée efficacement, quels sont les écueils à éviter ?). 
13 A la croisée des didactiques des disciplines et de la sémiologie de la bande dessinée, l'ambition du présent dossier était donc de considérer le médium dans toutes ses spécificités, et ainsi de clarifier ce qu'il est possible d'attendre de son exploitation, dans différents contextes d'enseignement. Loin d'engager une opération d'instrumentalisation ou de récupération de la bande dessinée au profit d'apprentissages variés sans rapport avec elle, cette réflexion sur les "usages didactiques de la bande dessinée » vise au contraire à interroger rigoureusement les enjeux du choix d'une bande dessinée comme support pédagogique.

De fait, les neufs articles et l'entretien proposés ici abordent une pluralité de disciplines (physique, sciences, histoire-géographie, sciences économiques et sociales, allemand, arts plastiques, lettres) et de niveaux d'enseignement (du premier degré au master).

Le choix a aussi été fait d'articuler des réflexions et retours d'expériences plus ou moins formalisés avec des résultats de recherche, afin tout à la fois de rendre de compte des dernières avancées de ce domaine et d'inviter à poursuivre la réflexion sur ces questions, à différentes échelles.

Les articles du dossier s'intéressant aux pratiques et représentations en vigueur sur la bande dessinée parmi les enseignants confirment que celle-ci reste un objet didactique assez neuf, autour duquel les pratiques ne sont pas stabilisées. Analysant un corpus de séquences de littérature publiées en ligne à destination des enseignants du primaire, Raux relève un certain mouvement d'intégration de bandes dessinées aux œuvres lues et travaillées en tant qu'œuvres intégrales; si les ressources disponibles sont peu nombreuses, elles suscitent un intérêt manifeste de la communauté enseignante dans le domaine. Le cadre de la lecture littéraire est cependant adapté avec difficultés aux spécificités du médium. L'article de Dardaillon et Meunier sonde quant à lui des étudiants se préparant au professorat des écoles: il ressort de cette enquête que ces futurs enseignants ne voient guère comment ils pourraient intégrer de la bande dessinée à leur enseignement, en raison surtout d'une méconnaissance assez nette du médium et de la diversité de ses productions. Formateurs d'enseignants, les auteurs présentent également des dispositifs visant à familiariser leurs étudiants avec le langage de la bande dessinée et à ouvrir des pistes pour que la narration visuelle entre dans les classes et contribue à l'appropriation de savoirs.

17 Le dossier invite ensuite à interroger la façon dont le langage de la bande dessinée travaille des savoirs, à travers plusieurs articles questionnant les enjeux de la réception et de la production de bandes dessinées à visée didactique. Lautesse et al. rendent compte d'une séance co-animée avec un philosophe des sciences et menée avec des étudiants de physique, pour réfléchir à certains enjeux de la mécanique quantique. Des vignettes d'une bande dessinée co-écrite par un physicien et un auteur de bande dessinée sont convoquées et questionnées, la mise en récit et en images de controverses scientifiques et de notions abstraites devant favoriser l'engagement des étudiants dans une réflexion épistémologique. Maron et al. présentent un protocole d'écriture de bandes dessinées didactiques numériques qui inscrivent des notions scientifiques dans l'histoire de leur découverte et dans les débats qui ont accompagné leur découverte. L'élaboration de ces œuvres, réalisées en partenariat entre des auteurs de bande dessinée et des didacticiens des sciences, s'appuie sur des tests de lecture dans des classes. C'est un de ces tests, portant sur l'épisode "gravitation", qui est ici analysé : en interrogeant précisément ce que des élèves de lycée ont compris et retenu de la notion à l'issue de la lecture, mais aussi comment ils ont perçu cette expérience de lecture d'une bande dessinée numérique, 
l'article examine comment s'articulent ces deux dimensions, et souligne l'importance de l'étayage à mettre en œuvre pour que les enjeux conceptuels soient bien saisis.

De manière complémentaire, la production de bandes dessinées par des élèves peut être envisagée comme une activité susceptible de travailler des savoirs. C'est l'objet de l'article de De Hosson et al., qui analyse la production de planches de bande dessinée de médiation scientifique réalisées par des collégiens au cours d'ateliers co-animés par des auteurs de bande dessinée et de jeunes chercheurs doctorants dans différentes disciplines. En travaillant des éléments d'un discours savant comme matière narrative, l'élève-auteur est amené à arbitrer entre effet recherché sur le lecteur (relevant souvent de l'humour) et rigueur dans la validité des connaissances en jeu. Les échanges concernant ces arbitrages au cours de l'atelier montrent que les choix effectués peuvent révéler une bonne assimilation des savoirs en même temps qu'une appropriation du système de la bande dessinée, ainsi transformée en instrument d'appropriation de connaissances. Dans son article, le dispositif que propose Ginoulhiac en arts plastiques au lycée rejoint sur plusieurs points cette expérience: des élèves de terminale, suivant cette discipline en spécialité, ont réalisé au cours d'un atelier avec un auteur de bande dessinée un strip à partir d'un tableau étudié dans la perspective de l'histoire des arts. L'analyse des productions montre de la même façon que l'effet bande dessinée sur les choix scénaristiques se donne à voir à travers une prédominance de l'humour, et que l'ensemble des propositions, dont la variété atteste d'un engagement créatif dans l'activité, associe diversement réinvestissement des connaissances acquises en amont sur l'œuvre et prise de distance avec le support initialement travaillé pour couler la création dans les conventions de la bande dessinée.

列 plusieurs disciplines au regard des compétences mobilisées par des situations de lecture variées. A partir d'un enseignement de sciences économiques et sociales en Première ES, Polo et Rouvière soulignent les risques qu'entraine le choix d'un récit en bande dessinée pris comme support d'une analyse sociologique sans analyse préalable de ses caractéristiques spécifiques et des compétences de lecture qu'il suppose. Lorsque les élèves sont soumis pour une évaluation à un extrait de bande dessinée dans lequel ils doivent identifier des notions sociologiques étudiées au préalable, ils peinent en majorité à mettre en relation le récit, en particulier les éléments portés par l'image, avec les notions attendues; quelques-uns commettent même des erreurs de lecture. Invités à un retour réflexif sur l'évaluation, les élèves mettent pourtant majoritairement en avant le caractère plaisant de l'activité : cette distorsion entre la perception positive du support et la réussite en demi-teinte de la tâche invite à examiner rigoureusement quelles compétences sont en jeu dans une activité mobilisant un support en bande dessinée.

Dans cette même perspective, Schnitzer et al. montrent que la complémentarité entre le texte et le dessin est à l'origine de situations-problèmes susceptibles de favoriser des acquisitions en langues. Elles s'appuient pour cela sur l'usage d'extraits d'un roman graphique et d'un strip humoristique en cours d'allemand au lycée. En histoire, l'article de Groeninger examine comment, grâce à l'humour dont ils sont empreints et à la distance que le dessin permet de prendre avec d'autres images plus frontales, des romans graphiques à caractère autobiographique pourraient constituer un support pertinent pour aborder la question sensible des conflits du Moyen-Orient, à condition de questionner et non de postuler leur caractère documentaire. 
21 Rouvière trace enfin des perspectives pour une didactique de la bande dessinée en classe de littérature. L'entretien proposé est l'occasion d'une synthèse des nombreux travaux de recherche dans lesquels il a analysé des dispositifs de lecture et de production de bandes dessinées dans des classes allant de l'école élémentaire au lycée.

22 A travers l'ensemble des articles, le dossier fait dialoguer réflexion didactique sur les enjeux en termes d'apprentissages d'activités impliquant la bande dessinée, et réflexion d'ordre sémiologique sur les spécificités du médium. Ce dialogue permanent invite à dépasser la dichotomie souvent relevée entre bande dessinée comme objet d'enseignement et comme support d'enseignement : un travail avec la bande dessinée se doit de comporter aussi un travail sur la bande dessinée, pour éviter à la fois une instrumentalisation réductrice du médium et de potentiels malentendus socio-cognitifs autour de supports qui ne seraient pas appréhendés dans toute leur richesse et leur complexité.

\section{BIBLIOGRAPHIE}

Aquatias, S. (2015). Le goût de la bande dessinée : acquisitions, transmissions, renforcements et abandons In La bande dessinée : quelle lecture, quelle culture ? Paris : Éditions de la Bibliothèque publique d'information. http://books.openedition.org/bibpompidou/1675. ISBN : 9782842462178. DOI : 10.4000/books.bibpompidou.1675.

Aquatias, S. (2017). La bande dessinée à l'école hier et aujourd'hui. Deuxièmes rencontres nationales de la bande dessinée, Angoulême. http://www.etatsgenerauxbd.org/wp-content/uploads/ sites/9/2018/05/2017_rencontres_nationales_bd_02_v00c2.pdf

Baudry, J. (2015). Jeune recherche : pluridisciplinarité innée ou acquise ? Les Carnets de Comicalités. https://graphique.hypotheses.org/632

Bautier, E., Crinon, J., Delarue-Breton, C. et Marin, B. (2012). Les textes composites : des exigences de travail peu enseignées ?, Repères, 45, 63-79.

Bautier, E. et Rocheix, J.-Y. (1997). Apprendre : des malentendus qui font la différence. In Terrail J. (dir.), La scolarisation de la France. Critique de l'état des lieux. Paris : La Dispute. Repris in Deauvieau J. et Terrail J.-P. (dir.) (2007). Les sociologues, l'école et la transmission des savoirs. Paris : La Dispute, p. 227-241.

Berthou, B. (2015). Bande dessinée, école et université : quelle mésentente ?, Les Carnets de Comicalités. https://graphique.hypotheses.org/537

Boltanski, L. (1975). La constitution du champ de la bande dessinée. Actes de la recherche en sciences sociales, vol. $1, \mathrm{n}^{\circ} 1,37-59$.

Bomel-Rainelli, B. et Demarco, A. (2011). La BD au collège depuis 1995 : entre instrumentalisation et reconnaissance d'un art. Le français aujourd'hui, 172(1), 81-92.

Bonnéry, S. (2007). Comprendre l'échec scolaire. Élèves en difficultés et dispositifs pédagogiques. Paris : La Dispute, 2007. 
Bonnéry S., Crinon, J., Marin, B. (2015). Des inégalités d'usage de la littérature de jeunesse dès les premiers cycles de l'école primaire? Une enquête par questionnaires. Spirale. Revue de recherches en éducation, $n^{\circ}$ 55, 43-56.

Boutin, J.-F. (2014). «Atouts et limites du texte illustré : développer l'interprétation avec les élèves à partir de la bande dessinée. » Québec français, (172), 72-74.

Dardaillon, S. (2009). Quelle place pour l'iconotexte dans les pratiques enseignantes au cycle III ? In Louichon, B. et Rouxel, A. (dir.), La littérature en corpus. Corpus implicites, explicites, virtuels. Dijon : SCEREN, CRDP de Bourgogne, 85-95.

Détrez, C. et Vanhée, O. (2012). Les mangados : lire des mangas à l'adolescence. Paris : Éditions de la Bibliothèque publique d'information, 2012.

Dupaire, C. (2019). Etat des lieux : la place de la bande dessinée dans l'enseignement. Syndicat national de l'Edition. https://www.sne.fr/document/etude-la-place-de-la-bande-dessinee-danslenseignement/

Groensteen, T. (2006). Un objet culturel non identifié : la bande dessinée. Mouthiers-Sur-Boëme, Éditions de l'An 2.

Groensteen, T. (2017). La bande dessinée au tournant. Editions de l'An 2.

Guilbert, W. (2011). La légitimation en devenir de la bande dessinée, Comicalités. http:// journals.openedition.org/comicalites/181

Lacelle, N. (2012). Des propositions d'enseignement de lecture littéraire et filmique pour fonder une didactique de la lecture multimodale. In Lebrun, M., Lacelle, N. et Boutin, J.-F. (dir), La littératie médiatique multimodale. De nouvelles approches en lecture-écriture à l'école et hors de l'école. Québec : Presses de l'université du Québec, 171-188.

Lapp, D., Wolsey, T.D., Fischer, D. , Frey, N. (2012). Graphic novels : what teachers think about their instructional value. Journal of Education, 192.

Lesage, S. (2018). La bande dessinée, entre mainstream et avant-gardes, Savoir/Agir, vol. 44, no. 2, 47-53.

Louichon, B. (2008). Enquête sur le rapport des enseignants aux programmes de 2002 et les pratiques d'enseignement de la littérature au cycle 3, Repères, 37, 51-68.

Mack, J. M. d. 2012. Histoire culturelle et bande dessinée : pistes méthodologiques et propositions pédagogiques pour questionner la BD en tant que document historique. In Rouvière, N. (dir.), Bande dessinée et enseignement des humanités. UGA Éditions. doi :10.4000/books.ugaeditions.1259

Maigret, É. (1994). La reconnaissance en demi-teinte de la bande dessinée. Réseaux, 67(5), 113-140.

Maigret, É. (2015). La bande dessinée dans le régime du divertissement : reconnaissance et banalisation d'une culture. In La bande dessinée : quelle lecture, quelle culture ? Paris : Éditions de la Bibliothèque publique d'information. http://books.openedition.org/bibpompidou/1677. DOI : 10.4000/books.bibpompidou.1677.

Maigret, Éric. La reconnaissance en demi-teinte de la bande dessinée, Réseaux, vol. 67, no. 5, 1994, 113-140. https://halshs.archives-ouvertes.fr/halshs-01172145/document

Méon, Jean-Matthieu. Bande dessinée : une légitimité sous conditions, Informations sociales, vol. 190, no. 4, 2015, 84-91.

Rosier, J. 2012. La BD à l'École : un leurre pédagogique en Belgique francophone. In Rouvière, N. (dir.), Bande dessinée et enseignement des humanités. UGA Éditions. doi :10.4000/

books.ugaeditions.1232 
Rouvière, N. (2012). Bande dessinée et enseignement des humanités. Grenoble : ELLUG. 7-18.

Rouvière, N. (2017). Perspectives de formation à la didactique de la bande dessinée en classe de littérature. Deuxièmes Rencontres nationales de la bande dessinée, Angoulême. http:// www.etatsgenerauxbd.org/wp-content/uploads/ sites/9/2018/05/2017_rencontres_nationales_bd_02_v00c2.pdf

Steyaert, F. et Tilleuil, J.-L. (2016). La bande dessinée à l'école. Un caillou dans le soulier de la légitimation, dans Ahmed, M., Delneste, S. et Tilleuil, J.-L. (dir.), Le statut culturel de la bande dessinée. Ambiguités et évolutions. Louvain-la-Neuve/Paris, Academia/L'Harmattan, Textes-Images, $n^{\circ} 8,233-268$.

\section{NOTES}

1. Des constats similaires sont faits dans d'autres contextes, des chercheurs notent par exemple la « frilosité » des programmes belges (Rosier, 2012 ; voir également Steyaert et Tilleuil, 2015) ou pointent que les programmes québécois sont «relativement silencieux» (Boutin, 2014) en matière de bande dessinée.

2. Sur ce point aussi des constats similaires sont faits dans d'autres contextes que la France : aux Etats-Unis par exemple, les enseignants, tout en se disant majoritairement intéressés par la bande dessinée, confient ne pas savoir comment l'aborder, selon Lapp et al. (2012).

3. Enquête menée par la Bibliothèque publique d'information (Service étude et recherche), avec le concours du Département des études, de la prospective et des statistiques du ministère de la Culture et réalisée par TMO Régions (voir http://neuviemeart.citebande dessinée.org/spip.php?article581 : consulté le 28 janvier 2018)

\section{AUTEURS}

\section{MARIANNE BLANCHARD}

Université de Toulouse, UT2J/Espe Midi-Pyrénées, CERTOP

HÉLÈNE RAUX

Université de Montpellier - LIRDEF 